\title{
Monte Carlo Simulation of Grain Growth
}

\author{
Paulo Blikstein, André Paulo Tschiptschin \\ Depto. de Eng. Metalúrgica e de Materiais, Escola Politécnica da Universidade de São Paulo \\ Av. Prof. Mello Moraes, 2463 São Paulo - SP, Brazil
}

Received: August 15, 1998; Revised: March 30, 1999

\begin{abstract}
Understanding and predicting grain growth in Metallurgy is meaningful. Monte Carlo methods have been used in computer simulations in many different fields of knowledge. Grain growth simulation using this method is especially attractive as

- the statistical behavior of the atoms is properly reproduced;

- microstructural evolution depends only on the real topology of the grains and not on any kind of geometric simplification.

Computer simulation has the advantage of allowing the user to visualize graphically the procedures, even dynamically and in three dimensions.

Single-phase alloy grain growth simulation was carried out by calculating the free energy of each atom in the lattice (with its present crystallographic orientation) and comparing this value to another one calculated with a different random orientation. When the resulting free energy is lower or equal to the initial value, the new orientation replaces the former. The measure of time is the Monte Carlo Step (MCS), which involves a series of trials throughout the lattice. A very close relationship between experimental and theoretical values for the grain growth exponent $(n)$ was observed.
\end{abstract}

Keywords: Monte Carlo simulation, grain growth, physical metallurgy, scientific visualization

\section{Introduction}

Grain size is a very important characteristic for evaluating the properties of the materials, especially when we need to balance different ones. It is, in fact, one of the most important issues in the control of microstructure ${ }^{1}$. As a result, this phenomenon is exhaustively studied in Metallurgy.

Usually this kind of study is made through careful analysis and comparison of microstructures, often with the help of image analysis software. Recently, as the processing power of computers increased a new and promising approach has been made possible: computer simulation of grain growth.

Srolovitz et al. ${ }^{2,3}$ proposed the most widely known and employed theory for computer modeling and simulation of grain growth, using the Monte Carlo method. There is a very interesting relationship between the conceptual basis of the Monte Carlo method and the physical characteristics of grain growth. As far as both rely very much on statistics and randomness, the method seems to represent the phenomenon very well.

ae-mail: pblikste@1si.usp.br

be-mail: aptschip@usp.br

\section{Grain Boundary Migration}

During grain growth the boundary equilibrium is reached when the angles among them follow the equation:

$$
\frac{\gamma_{1-2}}{\sin \alpha_{1}}=\frac{\gamma_{2-3}}{\sin \alpha_{2}}=\frac{\gamma_{1-3}}{\sin \alpha_{3}}
$$

where $\alpha_{n}$ are the angles between boundaries and $\gamma_{\mathrm{n}-\mathrm{m}}$ are the specific surface energies. In a single-phase alloy, considering a $2 \mathrm{D}$ simplified anisotropic structure, the equilibrium angle is $120^{\circ}$.

Burke ${ }^{4}$ proposed that the rate of boundary migration would be inversely proportional to the average curvature radius:

$$
\bar{R}=k t^{n}
$$

where $\overline{\mathrm{R}}$ is the grain diameter, $t$ is the time and $k$ is a constant that varies exponentially with temperature. The maximum value of the exponent $n$ (rarely observed experimentally) is 0.50 .

Equation 2, however, describes only an average growth. It cannot predict, for instance, different growth rates for the 
different grain size classes present in the microstructure. As a result, we conclude that there are very important issues that are simply not possible to evaluate without computer simulation. As mentioned by Srolovitz et al.:

"While it is generally observed that large grains grow and small grains shrink, instances where the opposite is true can be found. This suggests that knowledge of the instantaneous absolute or normalized grain sizes is not sufficient to predict the evolution, or even the direction of evolution, of individual grains. [...] The results indicate the validity of a random walk description of grain growth kinetics for large grains, and curvature driven kinetics for small grains."3

Computer simulation of grain growth plays an important role in understanding it. The goal is not simply to confirm the conventional theory, but to investigate new issues.

\section{Description of the Method}

The concept behind the Monte Carlo method in grain growth simulation is both simple and fascinating: its only basis is the thermodynamic of atomic interactions. There are no other experimental or theoretical inferences, nor mathematical approximations.

The first step is to represent the material as a $2 \mathrm{D}$ or $3 \mathrm{D}$ matrix, in which each site corresponds to a surface or volume element. The content of each element represents its crystallographic orientation. Contiguous regions (containing the same "number") represent the grains as shown in Fig. 1. The grain boundaries are fictitious surfaces that separate volumes with different orientations.

After choosing the kind of matrix and filling it with an initial random content, the simulation itself begins. These are the four main steps of the algorithm:

a) Calculation of the free energy of an element of the matrix $\left(G_{i}\right)$ with its present crystallographic orientation $\left(Q_{i}\right)$ based on its neighborhood.

b) Random choice of a new crystallographic orientation for that element $\left(\mathrm{Q}_{\mathrm{f}}\right)$.

c) New calculation of the free energy of the same element $\left(\mathrm{G}_{\mathrm{f}}\right)$, but with the new crystallographic orientation $\left(\mathrm{Q}_{\mathrm{f}}\right)$.

d) Comparison of the two values $\left(\mathrm{G}_{\mathrm{f}}-\mathrm{G}_{\mathrm{i}}\right)$. The orientation that minimizes the energy is chosen.

These four steps are repeated millions of times in random positions of the matrix. The overall result is a microscopic simulation of the free energy decay in the system, which is actually the main impelling force for grain growth. Figures 2 and 3 show schematically the four main steps applied to one element of the matrix. Figure 4 shows an example of energy calculation at a stable triple point where any reorientation attempt would lead to the same free energy value.

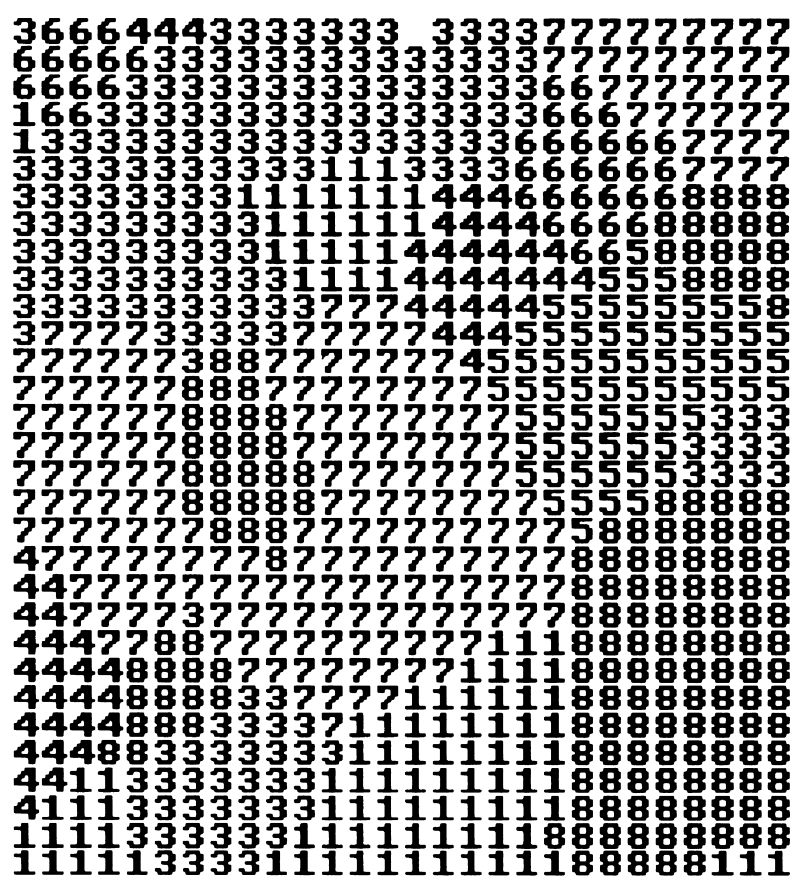

Figure 1. The grain structure represented by a 2D square matrix.

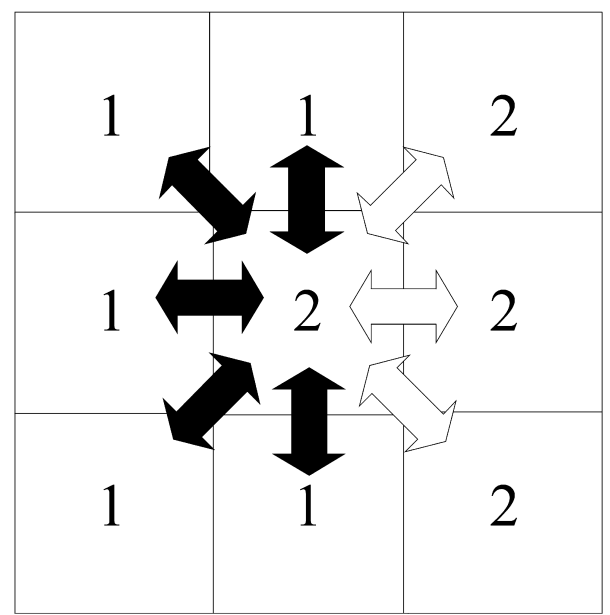

Figure 2. Calculation of the free energy of an element of the matrix $\left(\mathrm{Q}_{\mathrm{i}}\right.$ $=2$ ). In this example, $\mathrm{G}_{i}=5$ (five different neighbors).

The Hamiltonian that describes the interaction among the closest neighbors, which represents the grain boundary energy, is:

$$
H=-J \sum_{n n}\left(\delta_{S_{i} S_{j}}-1\right)
$$

where $\mathrm{Si}$ is one of the $Q$ possible orientations in the $i$ element of the matrix and $\delta_{a b}$ is the Kronecker-delta, which is $l$ when the two elements are equal and 0 otherwise. As a result, neighbors with a different orientation contribute $J$ to the system energy and $O$ when equal. The transition probability $W$ is: 


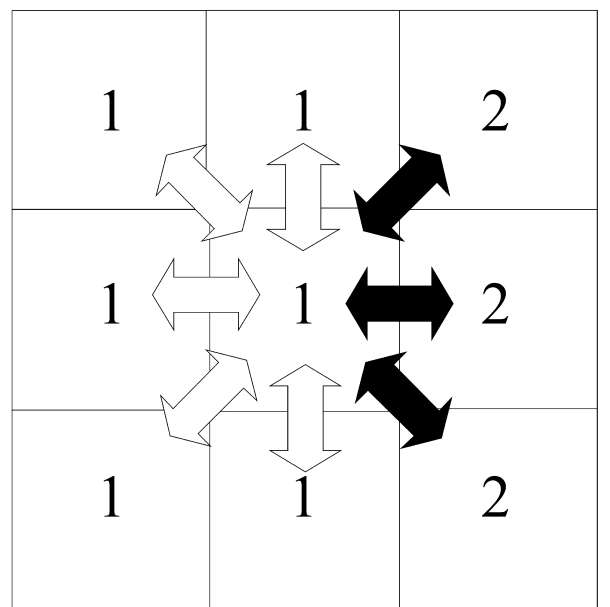

Figure 3. After an attempt of reorientation $\mathrm{To}_{\mathrm{Q}}=1$, the free energy is reduced to $\mathrm{G}_{\mathrm{f}}=3$. hence the NEW orientation should be maintained.

$$
\boldsymbol{W}=\left\{\begin{array}{l}
\exp \left(\frac{-\Delta G}{k_{b} T}\right) \rightarrow \Delta G>0 \\
1 \rightarrow \Delta \mathrm{G} \leq 0
\end{array}\right.
$$

where $\Delta \mathrm{G}$ is the change in free energy due to the orientation alteration, $k_{b}$ is the Boltzman constant and $T$ is the temperature. Thus the speed of the moving segment is given by:

$$
v_{i}=C\left[1-\exp \left(\frac{-\Delta G_{i}}{k_{b} T}\right)\right]
$$

where $\mathrm{C}$ is the boundary mobility.

\section{Evolutions of the Classical Method}

At first sight, the algorithm describes accurately the physical phenomena. Nevertheless, some authors noticed problems: nucleation of an "artificial" grain inside others

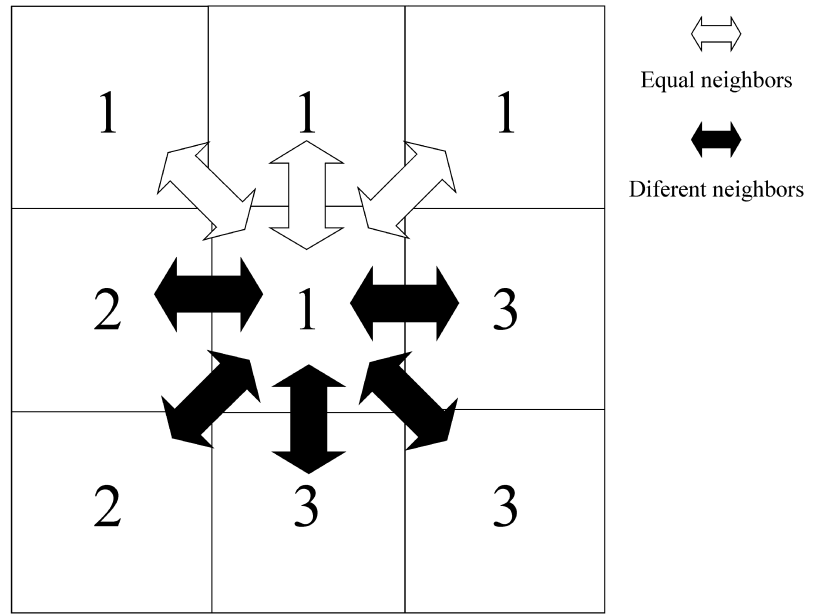

Figure 4. Stability of a triple point, with three $120^{\circ}$ angles. A reorientation attempt to $Q=3$ would lead to the same free energy value $(G=5)$. An attempt to $\mathrm{Q}=2$ would result in a greater value $(\mathrm{G}=6)$, not favorable. Hence this configuration is stable, unless for statistical fluctuations. (which does not occur experimentally) and values of $n$ below 0.50 (the theoretical value of the grain growth exponent). Radhakrishnan e Zacharia ${ }^{6}$ proposed a new algorithm that would at one time eliminate artificial nucleation, raise the value of $n$ and decrease significantly the computing time required for the simulation.

The new algorithm proposes that the new orientation to be "evaluated" in each matrix element would no more be chosen among all the possible ones (Q-1), but only among those that surround each element. In other words, if an element has in its surroundings grains with orientations 3 , 6,13 and 15 , the new one will be chosen only among these four values.

This was the algorithm used in our experiments, also called "cellular automata".

\section{Experimental}

The software was developed using a $\mathrm{C}++$ compiler. The program was executed in a Pentium-Pro $233 \mathrm{MHz}$ machine with $128 \mathrm{MB}$ of RAM. The machine showed up to be very satisfactory in terms of speed and reliability for 2D simulations.

The visualization of the matrix was carried out through conventional image processing software. The $3 \mathrm{D}$ visualization (which is still being improved) was done in a Silicon Graphics workstation running the $A V S$ visualization package.

\section{Results in a 2D Square Lattice}

The experiments took place in a $200 \times 200$ matrix. The dimensional evolution of the grains was measured by evaluating the total amount of interface in the system. In Fig. 5 we can observe the microstructure after some simulation steps.

The first important issue to be analyzed is the geometry (square or triangular) of the matrix to be used. Srolovitz et $a l .{ }^{1}$ analyzed this problem and reached the conclusion that the results were equivalent, provided higher temperatures were used in the simulations with square matrix. This is due to the fact that in this kind of discretization, a perfect $120^{\circ}$ angle between boundaries in not possible. In the triangular lattice, the temperature can be set to $\mathrm{T}=0$ as its geometric nature can represent with more accuracy the triple points and grain boundaries. Temperature, in all these Monte Carlo simulations, is not directly related to "real" temperature. It is just a correction factor for the different characteristics of the lattices. Some authors introduced "real" temperature gradients in the simulation model by changing boundary mobility along the matrix ${ }^{7}$.

The second important issue is the maximum number of possible orientations (Q). The first experiments by Srolovitz et al. ${ }^{2}$ employed values from 4 to 64 , while Radhakrishnan ${ }^{6}$ proposed $\mathrm{N}^{2}$, where $\mathrm{N}$ is the dimension of the matrix (in a $200 \times 200$ matrix, $Q=200^{2}=40,000$ ), as 


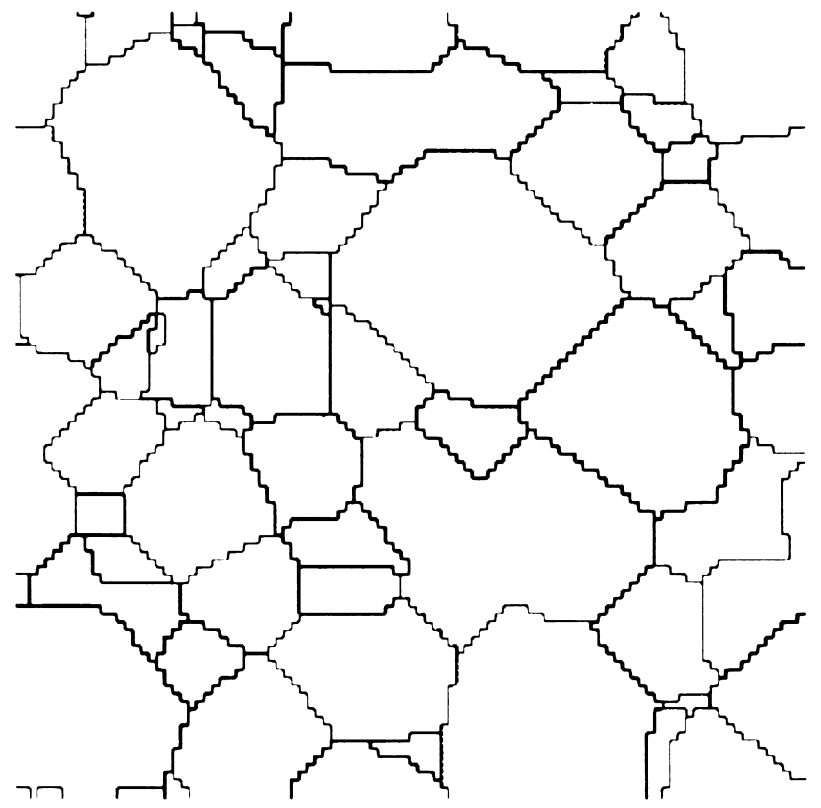

Figure 5. The microstructure after editing in image processing software.

to avoid artificial grain coalescence. We ran nine series of simulations, with values of $Q$ from 8 to 30,000. The results are shown in Fig. 6. It is clearly noticeable that the value of $\mathrm{Q}$ alters the kinetics of grain growth. For the lowest value (8) the grain growth exponent is the highest $(0.54)$, probably due to artificial grain coalescence. However, much higher $Q$ values $(256,512,1024,30000)$ did not improve significantly the results, which were very similar to those obtained in the simulations with $\mathrm{Q}=64$ or $\mathrm{Q}=128$. Srolovitz et al. ${ }^{2}$ performed a similar experiment and concluded that for $\mathrm{Q}>30$ the simulation becomes Q-independent. Based on these results a $Q$ value of 128 was chosen for the subsequent experiments.

After these initial experiments, we proceeded to grain growth investigation itself. The conventional algorithm proposes that the new orientation should be chosen at random among all possible orientations ( $Q-1)$. Experiments obtained with this algorithm ${ }^{2,3,6}$ reached values for the grain growth exponent $n$ still far from the theoretical value

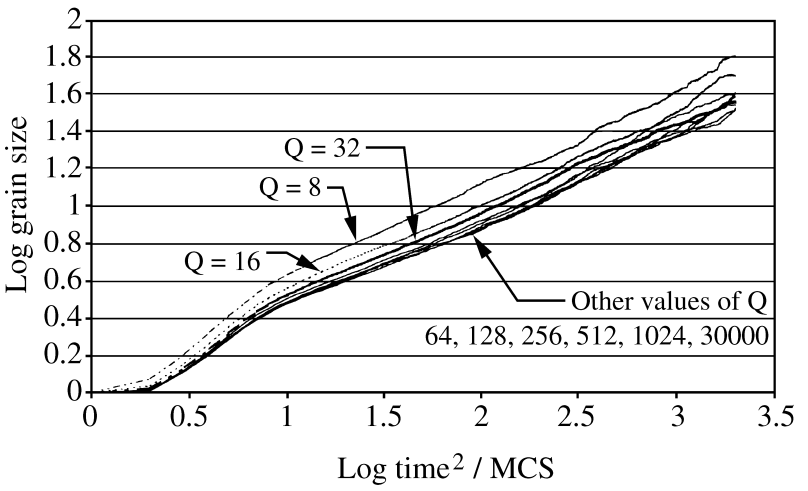

Figure 6. Grain growth behavior for increasing q values.
(0.50). Radhakrishnan demonstrated that the kinetics of the conventional method would lead to a significantly slower microstructural evolution, and proposed a new one, which is widely employed nowadays.

To investigate this issue, we ran two series of simulations, with the old and new algorithm. Fig. 7 shows the results. There was a clear difference between the two simulations, both in the growth kinetics and the final microstructure. Using the old algorithm, we reached values for $n$ within the $0.35-0.39$ range. Employing the new algorithm, the values were in the $0.48-0.51$ range, much closer to the theoretical 0.50 .

After averaging a series of simulations with the new algorithm, in a square matrix of $200 \times 200$, the value of 0.509 was reached. We also proceeded a careful statistical analysis of the local slopes of the grain growth curve. As expected, the histogram shown in Fig. 8 revealed that most of the local slopes were within the $0.40-0.60$ range. As the simulations relies very much on random numbers, it is not surprising that there are negative slopes $(-0.60)$ as well as much higher ones $(+1.60)$. The average, however, was very close to what was expected (0.50).

In the $\log x \log$ scale of the graph in Fig. 9 it is possible to notice the linear behavior of the average curve. In very long simulations (> 50,000 MCS) we noticed that the system reaches stability, with a very small number of grains. Reaching a sufficiently large size, their growth slows down, as most of the boundaries become flat (in a square matrix). The values of $n$ found in the simulation are in very good agreement with the experiments of various

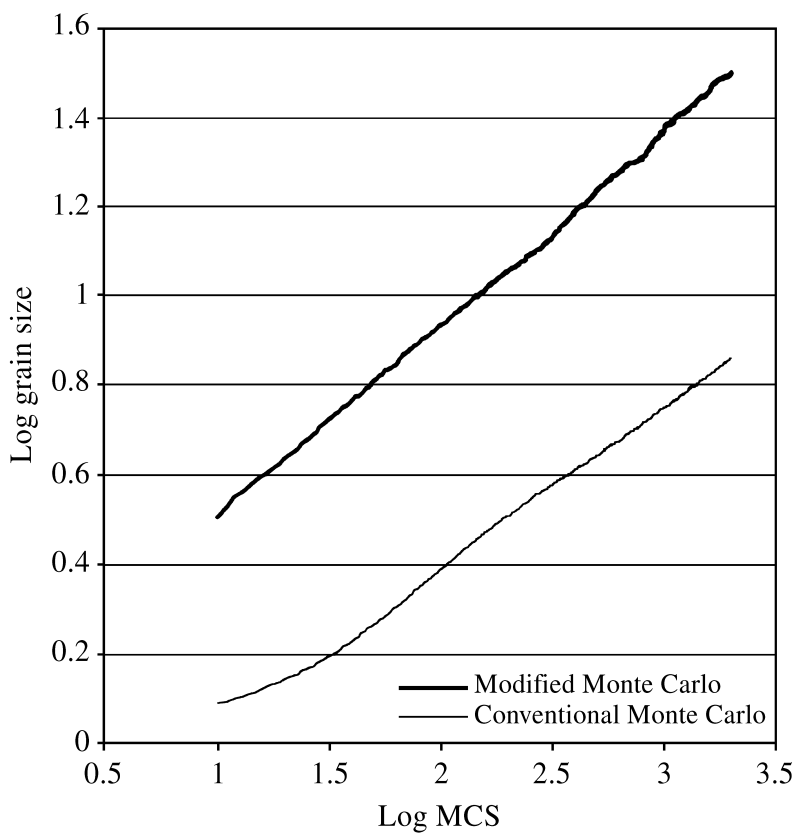

Figure 7. Grain growth behavior with the conventional and modified methods. while $n$ values found for the old method were around 0.35 , values of 0.49 were reached for the new algorithm. 


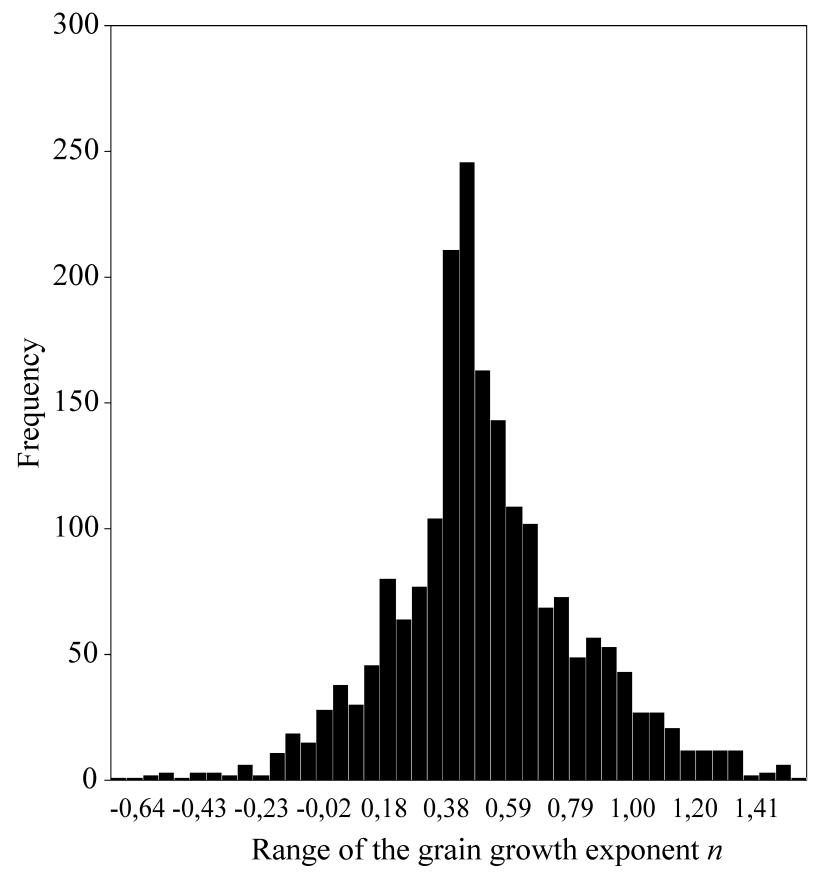

Figure 8. Histogram of the local slopes.

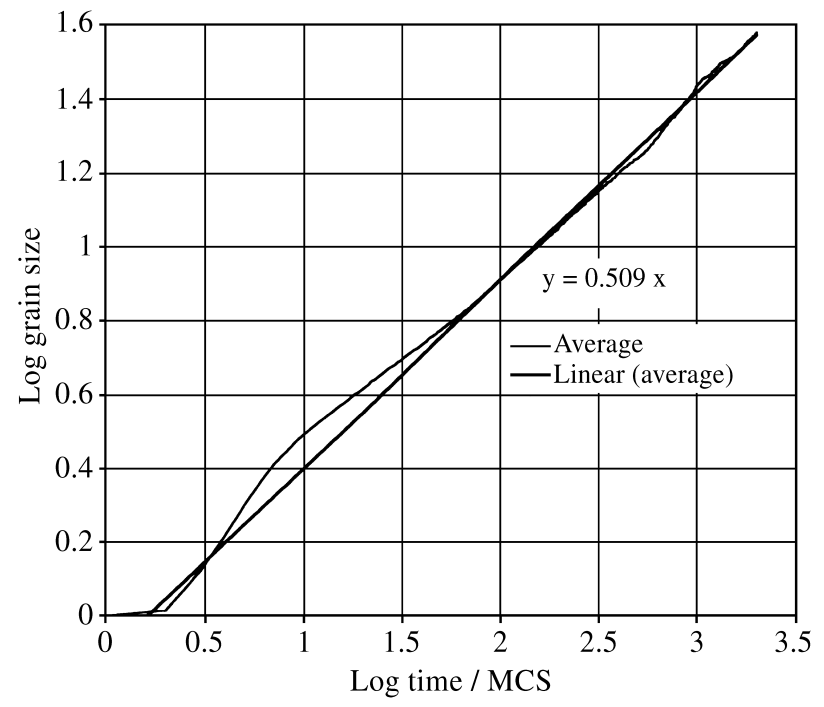

Figure 9. Grain size $v s$. time (5000 mcs).

authors, as Srolovitz et al. ${ }^{2,3}$, Zacaria ${ }^{6}$ and Mehnert ${ }^{8,9}$. That demonstrates the validity of the software elaborated for the simulation and the model adopted.
The method discussed in this paper can be used in Metallurgy to study real-world problems, as the microstructure evolution during the welding process ${ }^{10}$ and grain growth pinning in advanced materials.

\section{Conclusion}

The computational simulation demonstrated that the grain growth phenomenon, although complex, could be modeled in a very elementary way. The correct value of $n$, found in our experiments, indicate that there is a very close relationship between the model and the theoretical description of grain growth.

\section{References}

1. Liu, Y.; Baudin, T.; Penelle P. "Simulation of normal grain growth by cellular automata". Scripta Materialia, v. 32, n. 11, p. 1679-1683, 1996.

2. Anderson, M.P.; Srolovitz, D.J.; Grest, G.S.; Sahni P.S. "Computer simulation of grain growth" - I. Kinetics”, Acta Metallurgica, n. 32, p. 783-792, 1984.

3. Anderson, M.P.; Srolovitz, D.J.; Grest, G.S.; Sahni, P.S. "Computer simulation of grain growth - II. Grain size distribution, topology and local dynamics", Acta Metallurgica n. 32, p. 793-801, 1984.

4. Burke, J.E. Trans. Am. Inst. Min. Engrs, n. 180, p. 73, 1949.

5. Saito, Y.; Enomoto, M. "Monte Carlo simulation of grain growth". ISIJ International, v. 32, n. 3, p. 267274 1992,.

6. Radhakrishnan, B.; Zacharia, T. "Simulation of curvature-driven grain growth by using a modified Monte Carlo algorithm". Metall. And Mater. Trans. A, n. 26A, , p. 167, 1995.

7. Miodownik, M.A. Sandia National Labs, personal communication, 1998.

8. Mehnert, K.; Klimanek. P. "Monte Carlo simulation of grain growth in textured metals". Scripta Materialia, v. 30, n. 6, p. 699-704, 1996.

9. Mehnert, K.; Klimanek, P. "Grain growth in metals with strong textures: three-dimensional Monte Carlo simulations". Computational Materials Science, 1997.

10. Fye, Richard. Sandia National Labs, personal communication, 1998. 